\title{
Supplementary data: Preoperative nutritional factors and outcomes after radical cystectomy: A narrative review
}

\author{
Janie Allaire ${ }^{1,2,3}$; Tal Ben-Zvi, MD 1,2; Benoît Lamarche, PhD; Karine Robitaille, PhD'; Yves Fradet, MD ${ }^{1,2}$; \\ Louis Lacombe, $M D^{1,2}$; Vincent Fradet, $M D^{1,2,3}$
}

'Department of Surgery, Université Laval; ${ }^{2}$ Centre de recherche du CHU de Québec - Université Laval, L'Hôtel Dieu de Québec; ${ }^{3}$ Institute of Nutrition and Functional Foods, Université Laval; Quebec, QC, Canada

Published online November 1, 2017

\begin{tabular}{|c|c|c|c|c|c|c|}
\hline First author & Year & $\begin{array}{l}\text { Eligible } \\
\text { subjects } \\
\quad \text { (n) }\end{array}$ & $\begin{array}{l}\text { Factor(s) of } \\
\text { nutritional } \\
\text { evaluation }\end{array}$ & Outcome & Results & $\begin{array}{l}\text { Methods of } \\
\text { classification } \\
\text { for } \\
\text { complications }\end{array}$ \\
\hline Hollenbeck ${ }^{26}$ & 2006 & 2538 & $\begin{array}{c}\text { Albuminemia } \\
\text { and } \geq 10 \% \text { weight } \\
\text { loss }\end{array}$ & $\begin{array}{l}\text { Length of hospital stay } \\
\text { (>30d) and mortality }\end{array}$ & $\begin{array}{l}\text { Logistic regression: Length of stay } \geq 30 \mathrm{~d} \text { : } \\
\text { albuminemia } \leq 3.5 \mathrm{~g} / \mathrm{dl} \text { (OR } 2.1 ; 95 \% \mathrm{Cl} \\
1.2-3.8 \text { ). Mortality within } 30 \mathrm{~d} \text { : weight } \\
\text { loss } \geq 10 \% \text { (OR } 2.7 ; 95 \% \mathrm{Cl} 1.1-6.4), 90 \mathrm{~d} \text { : } \\
\text { albuminemia } \leq 3.5 \mathrm{~g} / \mathrm{dl},(\mathrm{OR} 12.0 ; 95 \% \mathrm{Cl} \\
2.8-51.0 \text { ), weight loss } \geq 10 \% \text { (OR } 2.9 ; \\
95 \% \mathrm{Cl} 1.5-5.4)\end{array}$ & \\
\hline Reyes $^{33}$ & 2006 & 343 & BMI & $\begin{array}{c}\text { Neobladder-related } \\
\text { outcomes, pyelonephritis, } \\
\text { incisional hernia, wound } \\
\text { infections, wound } \\
\text { dehiscence, and voiding } \\
\text { patterns }\end{array}$ & $\begin{array}{l}\text { Chi-Square test: Normal weight vs. } \\
\text { overweight vs. obese subjects: Overall } \\
\text { complications ( } p=0.012) \text {, urinary tract } \\
\text { infection ( } p=0.001) \text {, pyelonephritis } \\
\text { ( } p=0.04) \text {, wound infection }(p=0.04 \text { ) were } \\
\text { less frequent in the normal weight group }\end{array}$ & \\
\hline Butt $^{34}$ & 2008 & 51 & BMI & $\begin{array}{l}\text { Complications rate, } \\
\text { hospital length of stay, } \\
\text { mortality }\end{array}$ & $\begin{array}{l}\text { Kruskal-Wallis and Fisher's exact test: } \\
\text { Hospital length of stay (results not } \\
\text { provided), complications rate (NS), } \\
\text { mortality (only one event) }\end{array}$ & \\
\hline Maurer ${ }^{35}$ & 2010 & 390 & BMI & $\begin{array}{l}\text { Complications and } \\
\text { mortality within } 90 \mathrm{~d} \text { after } \\
\mathrm{RC}\end{array}$ & $\begin{array}{l}\text { Mann-Whitney test: Higher BMI: } 30 \mathrm{~d} \\
\text { postoperative bleeding rate ( } \mathrm{p}=0.02 \text { ), } \\
\text { other complications (NS), mortality (NS). } \\
\text { Cox regression: Mortality (NS) }\end{array}$ & \\
\hline Novara $^{13}$ & 2009 & 358 & BMI & $\begin{array}{l}\text { All complications and } \\
\text { high-grade complications } \\
\text { (including mortality) } \\
\text { within } 90 \mathrm{~d} \text { after RC }\end{array}$ & NS & $\begin{array}{l}\text { MSKCC and } \\
\text { Clavien-Dindo }\end{array}$ \\
\hline Shabsigh $^{3}$ & 2009 & 1320 & BMI & $\begin{array}{l}\text { All complications and } \\
\text { high-grade complications } \\
\text { within } 90 \mathrm{~d} \text { after RC }\end{array}$ & NS & $\begin{array}{l}\text { MSKCC and } \\
\text { Clavien-Dindo }\end{array}$ \\
\hline Svatek $^{14}$ & 2009 & 283 & BMI & $\begin{array}{l}\text { Any AE within } 90 \mathrm{~d} \text { after } \\
\text { RC }\end{array}$ & $\begin{array}{l}\text { Logistic regression: AE 90d: BMI (OR } \\
\text { 1.16; } 95 \% \mathrm{Cl} 1.08-1.23) \text {. High-grade (2-4): } \\
\text { BMI (OR 1.10; 95\% Cl 1.04-1.16) }\end{array}$ & $\begin{array}{c}\text { Common } \\
\text { Terminology } \\
\text { Criteria } \\
\text { Adverse } \\
\text { Events }\end{array}$ \\
\hline
\end{tabular}

AE: adverse event; BMl: body mass indexd: Cl: confidemce interval; day(s); HR: hazard ratio; OR: odds ratio; MSKCC: Memorial Sloan Kettering Cancer Classification; Nb: number; NRS: nutritional risk screening; NS: non-statistically significant; RC: radical cystectomy; SMI: skeletal muscle index. 


\begin{tabular}{|c|c|c|c|c|c|c|}
\hline \multicolumn{7}{|c|}{ Supplementary Table 1 (cont'd). Summary of the studies included in this narrative review } \\
\hline First author & Year & $\begin{array}{l}\text { Eligible } \\
\text { subjects } \\
\quad \text { (n) }\end{array}$ & $\begin{array}{l}\text { Factor(s) of } \\
\text { nutritional } \\
\text { evaluation }\end{array}$ & Outcome & Results & $\begin{array}{l}\text { Methods of } \\
\text { classification } \\
\text { for } \\
\text { complications }\end{array}$ \\
\hline Svatek ${ }^{36}$ & 2010 & 283 & BMI & lleus & $\begin{array}{l}\text { Fisher's exact test: }(p=0.014) \\
\text { Logistic regression: (OR 1.09; } \\
\qquad 95 \% \mathrm{Cl} 1.03-1.17)\end{array}$ & \\
\hline Gregg $^{46}$ & 2011 & 905 & $\begin{array}{l}\text { Nutritional risk } \\
\text { was defined as } \\
\text { one or more } \\
\text { factor between: } \\
\text { albuminemia } \\
<3.5 \mathrm{~g} / \mathrm{dl} \text {, } \\
\mathrm{BMl}<18.5 \text {, and } \\
>5 \% \text { weight loss }\end{array}$ & $\begin{array}{l}\text { Overall mortality, } \\
\text { mortality within and after } \\
\text { 90d after RC }\end{array}$ & $\begin{array}{l}\text { Cox regression: Overall survival (HR 1.82; } \\
\text { 95\% Cl 1.25-2.65), within 90d (HR 2.91; } \\
95 \% \mathrm{Cl} 1.36-6.23) \text {, after 90d (HR 1.55; } \\
95 \% \mathrm{Cl} 1.01-2.38)\end{array}$ & \\
\hline Morgan $^{47}$ & 2011 & 220 & Albuminemia & $\begin{array}{l}\text { Mortality } 90 \mathrm{~d} \text { after } \mathrm{RC} \text { in } \\
\geq 75 \text { years old subjects }\end{array}$ & $\begin{array}{c}\text { Cox regression: Albuminemia }<3.7 \mathrm{~g} / \mathrm{dl} \\
\text { (HR 2.50; } 95 \% \mathrm{Cl} 1.40-4.45)\end{array}$ & \\
\hline Lambert ${ }^{49}$ & 2012 & 238 & Albuminemia & $\begin{array}{l}\text { Complications and overall } \\
\text { and cancer-specific } \\
\text { mortality after RC }\end{array}$ & $\begin{array}{l}\text { 2-sample test: Overall complications: } \\
\text { Albuminemia }<3.5 \mathrm{~g} / \mathrm{dl} \text { vs. normal } \\
\text { albuminemia ( } \mathrm{p}=0.014 \text { ). Cox regression: } \\
\text { Overall survival: Albuminemia } \\
<3.5 \mathrm{~g} / \mathrm{dl} \text { (HR } 1.76 ; p=0.04 \text { ), cancer- } \\
\text { specific survival: NS }\end{array}$ & \\
\hline $\operatorname{Large}^{37}$ & 2012 & 91 & BMI & Delirium & NS & \\
\hline Berger $^{15}$ & 2013 & 256 & BMI & $\begin{array}{l}\text { Inpatient complication } \\
\text { and mortality within } 90 \mathrm{~d}\end{array}$ & $\begin{array}{l}\text { Logistic regression: Inpatient } \\
\text { complications (continuously, OR 1.13; } \\
95 \% \mathrm{Cl} 1.02-1.24), \text { mortality (NS). } \\
\text { Log-rank test: mortality (NS) }\end{array}$ & Clavien-Dindo \\
\hline Chan $^{50}$ & 2013 & 117 & Albuminemia & $\begin{array}{c}\text { Overall mortality rate at } 5 \\
\text { years after } \mathrm{RC}\end{array}$ & $\begin{array}{l}\text { Cox regression: Albuminemia }>3.9 \mathrm{~g} / \mathrm{dl} \\
\text { (HR 0.946; } 95 \% \mathrm{Cl} 0.902-0.992 ; p=0.022 \text { ) }\end{array}$ & \\
\hline Chromecki $^{30}$ & 2013 & 4118 & BMI & $\begin{array}{l}\text { Cancer-specific and } \\
\text { overall mortality }\end{array}$ & $\begin{array}{c}\text { Cox regression: } \mathrm{BMI}>30 \mathrm{~kg} / \mathrm{m}^{2}: \text { Cancer- } \\
\text { specific mortality (HR } 1.43 ; 95 \% \mathrm{Cl} \\
1.24-1.66 ; \mathrm{p}<0.001 \text { ), overall mortality (HR } \\
1.81 ; 95 \% \mathrm{Cl} 1.60-2.05 ; \mathrm{p}<0.001 \text { ). Similar } \\
\text { results for } \mathrm{BMl} \text { as a continuous variable } \\
\text { (all } \mathrm{p} \text { values }<0.001 \text { ) }\end{array}$ & \\
\hline Djaladat ${ }^{48}$ & 2013 & 1964 & Albuminemia & $\begin{array}{l}\text { Any AE leading to } \\
\text { lengthening hospital } \\
\text { stay or re-admission } \\
\text { occurring within } 90 \mathrm{~d} \text { after } \\
\mathrm{RC} \text {, mortality within } 90 \mathrm{~d} \\
\text { recurrence-free survival, } \\
\text { and overall survival at } 5 \\
\text { years }\end{array}$ & $\begin{array}{l}\text { Logistic regression: Complications within } \\
90 \mathrm{~d} \text { (NS), mortality within } 90 \mathrm{~d} \\
\text { (few events } \mathrm{n}=15 \text { ) } \\
\text { Cox regression: Recurrence free survival } \\
\text { (HR } 1.68 ; 95 \% \mathrm{Cl} 1.16-2.43 \text { ), overall } \\
\text { survival (HR } 1.93 ; 95 \% \mathrm{Cl} 1.43-2.63 \text { ) }\end{array}$ & \\
\hline Jensen $^{54}$ & 2013 & 82 & NRS & $\begin{array}{l}\text { Length of hospital stay } \\
\qquad(\geq 11 \mathrm{~d})\end{array}$ & NS & \\
\hline Mursi ${ }^{16}$ & 2013 & 31 & $\begin{array}{l}\text { Albuminemia, } \\
\text { BMI }\end{array}$ & $\begin{array}{l}\text { Early ( } \leq 30 \mathrm{~d}) \text {, late ( } 31 \text { to } \\
90 \mathrm{~d}) \text { and cumulative } \\
(<90 \mathrm{~d}) \text { re-admission rate, } \\
\text { complications rate and } \\
\text { grade, and mortality after } \\
\text { RC }\end{array}$ & $\begin{array}{l}\text { Chi-Square: albuminemia }<3.5 \mathrm{~g} / \mathrm{dl} \text { : } \\
\text { higher mortality rate }(\mathrm{p}=0.048, \text { but few } \\
\text { events), NS for other outcomes. BMI: NS }\end{array}$ & Clavien-Dindo \\
\hline Xylinas ${ }^{31}$ & 2013 & 231 & BMI & Cancer-specific mortality & $\begin{array}{l}\text { Cox regression: Modelled continuously } \\
\text { (HR 1.50; } 95 \% \mathrm{Cl} 0.99-2.24 ; \mathrm{p}=0.052 \text { ) }\end{array}$ & \\
\hline
\end{tabular}

AE: adverse event; BMI: body mass indexd: Cl: confidemce interval; day(s); HR: hazard ratio; OR: odds ratio; MSKCC: Memorial Sloan Kettering Cancer Classification; Nb: number; NRS: nutritional risk screening; NS: non-statistically significant; RC: radical cystectomy; SMI: skeletal muscle index. 


\begin{tabular}{|c|c|c|c|c|c|c|}
\hline \multicolumn{7}{|c|}{ Supplementary Table 1 (cont'd). Summary of the studies included in this narrative review } \\
\hline First author & Year & $\begin{array}{l}\text { Eligible } \\
\text { subjects } \\
\text { (n) }\end{array}$ & $\begin{array}{l}\text { Factor(s) of } \\
\text { nutritional } \\
\text { evaluation }\end{array}$ & Outcome & Results & $\begin{array}{l}\text { Methods of } \\
\text { classification } \\
\text { for } \\
\text { complications }\end{array}$ \\
\hline Al-Daghmin 39 & 2014 & 272 & BMI & $\begin{array}{l}30 \text { and } 90 \mathrm{~d} \text { re-admission } \\
\text { rate }\end{array}$ & $\begin{array}{l}\text { Logistic regression: } 30 \mathrm{~d} \text { (OR 1.12; } \\
95 \% \mathrm{Cl} 1.05-1.19 ; \mathrm{p}=0.004) .90 \mathrm{~d} \text { (OR 1.10; } \\
95 \% \mathrm{Cl} 1.0-1.17 ; \mathrm{p}=0.004)\end{array}$ & \\
\hline Bachir $^{32}$ & 2014 & 847 & BMI & $\begin{array}{c}\text { Overall survival and } \\
\text { disease-specific survival }\end{array}$ & $\begin{array}{l}\text { Kaplan-Meier: No differences in overall } \\
\text { survival }(p=0.32) \text { and disease-specific } \\
\text { survival }(p=0.35) \text { between/among BMI } \\
\text { subgroups }\left(<25,25-29 \text {, and } \geq 30 \mathrm{~kg} / \mathrm{m}^{2}\right)\end{array}$ & \\
\hline Donahue ${ }^{45}$ & 2014 & 386 & $\begin{array}{l}\text { Albuminemia, } \\
\text { BMI }\end{array}$ & $\begin{array}{l}\text { Parastomal hernia within } \\
2 \text { years after RC }\end{array}$ & $\begin{array}{l}\text { Cox regression: Albuminemia } \\
\text { (continuously, HR } 0.43 ; 95 \% \mathrm{Cl} 0.25-0.75 \\
\text { p<0.003). BMI (continuously, HR 1.08; } \\
95 \% \mathrm{Cl} 1.05-1.12 ; \mathrm{p}<0.0001 \text { ) }\end{array}$ & \\
\hline $\operatorname{Garg}^{20}$ & 2014 & 1320 & Albuminemia & $\begin{array}{l}\text { Complications within } 30 \\
\text { and } 90 \mathrm{~d} \text { and mortality at } \\
90 \mathrm{~d} \text { after RC }\end{array}$ & $\begin{array}{l}\text { Fisher's exact test: Albuminemia } \\
<4 \text { vs } \geq 4 \mathrm{~g} / \mathrm{dl} \text { : Complications at } 30 \mathrm{~d} \text { : } \\
\text { neurological }(\mathrm{p}=0.001) \text {, wound } \\
\text { ( }<<0.001 \text { ), any complication ( } \mathrm{p}=0.005) \text {. } \\
\text { Complications between } 60 \text { and } 90 \mathrm{~d} \text { (NS). } \\
\text { Logistic regression: Grade } 1-5 \\
\text { complications within } 90 \mathrm{~d} \text { : (continuously, } \\
\text { OR } 0.61 ; 95 \% \mathrm{Cl} 0.42-0.90 \text { ). Mortality } \\
\text { within } 90 \mathrm{~d} \text { : (continuously, OR } 0.33 ; \\
95 \% \mathrm{Cl} 0.14-0.75 \text { ) }\end{array}$ & $\begin{array}{l}\text { MSKCC and } \\
\text { Clavien-Dindo }\end{array}$ \\
\hline Gandaglia ${ }^{21}$ & 2014 & 1094 & $\begin{array}{l}\text { Albuminemia, } \\
\text { BMI }\end{array}$ & $\begin{array}{l}\text { Complications within } 30 \mathrm{~d} \\
\text { (overall complications, } \\
\text { prolonged operative time, } \\
\text { prolonged length of stay, } \\
\text { perioperative mortality) }\end{array}$ & $\begin{array}{l}\text { Logistic regression: Overall complication: } \\
\text { Albuminemia: unknown vs. } \geq 3 \mathrm{~g} / \mathrm{dl} \text { (OR } \\
0.64 ; 96 \% \mathrm{Cl} 0.48-0.85 ; \mathrm{p}=0.01) \text {. BMI >30 } \\
\text { vs. }<25 \mathrm{~kg} / \mathrm{m}^{2} \text { (OR } 1.67 ; 95 \% \mathrm{Cl} 1.16-2.42 ; \\
\quad \mathrm{p}=0.01 \text { ). Other outcomes: NS }\end{array}$ & \\
\hline Johnson ${ }^{22}$ & 2014 & 1213 & $\begin{array}{l}\text { Albuminemia, } \\
>10 \% \text { weight } \\
\text { loss within } 6 \\
\text { months before } \\
\text { RC and BMI }\end{array}$ & $\begin{array}{c}\text { Complications within 30d } \\
\text { after RC }\end{array}$ & $\begin{array}{l}\text { Logistic regression: Albuminemia } \\
<3.5 \text { g/dl (OR 1.79; } 95 \% \mathrm{Cl} 1.06-3.03 \text { ). } \\
\text { BMI: NS. Weight loss: NS }\end{array}$ & \\
\hline Lavallée $e^{23}$ & 2014 & 2303 & $\begin{array}{l}\text { Albuminemia, } \\
>10 \% \text { weight } \\
\text { loss within } 6 \\
\text { months before } \\
\text { RC and BMI }\end{array}$ & $\begin{array}{c}\text { Complications within } 30 \mathrm{~d} \\
\text { after RC }\end{array}$ & NS & \\
\hline Psutka $^{41}$ & 2014 & 262 & $\begin{array}{l}\text { BMI and fat } \\
\text { mass index }\end{array}$ & Overall survival & $\begin{array}{l}\text { Cox regression: Increasing BMI } \\
\text { correlated with improved overall survival } \\
(p=0.03) \text {, fat mass index (NS) }\end{array}$ & \\
\hline Roghmann ${ }^{17}$ & 2014 & 535 & BMI & $\begin{array}{l}\text { All complications and } \\
\text { high-grade complications } \\
\text { within } 90 \mathrm{~d} \text { after RC }\end{array}$ & $\begin{array}{l}\text { Logistic regression: Any complication } \\
\text { (OR 1.08; 95\% Cl 1.03-1.13). High-grade } \\
\text { (3 and 4, OR 1.07; 95\% Cl 1.02-1.12) }\end{array}$ & $\begin{array}{l}\text { MSKCC and } \\
\text { Clavien-Dindo }\end{array}$ \\
\hline Tyson 24 & 2014 & 1293 & BMI & $\begin{array}{l}\text { 30d outcomes after RC: } \\
\text { mortality, wound events, } \\
\text { sepsis, pulmonary } \\
\text { events, renal failure, } \\
\text { thromboembolic and } \\
\text { cardiac events, hospital } \\
\text { length of stay, rates of } \\
\text { return to operating suite, } \\
\text { total operative time, and } \\
\text { total blood transfusions }\end{array}$ & $\begin{array}{l}\text { Fisher's exact test: } \mathrm{BMI}<30 \mathrm{vs} \text {. } \\
\geq 30 \mathrm{~kg} / \mathrm{m}^{2} \text { : operative time }(\mathrm{p}=0.04) \text {, NS } \\
\text { for other outcomes }\end{array}$ & \\
\hline
\end{tabular}

AE: adverse event; BMI: body mass indexd: Cl: confidemce interval; day(s); HR: hazard ratio; OR: odds ratio; MSKCC: Memorial Sloan Kettering Cancer Classification; Nb: number; NRS: nutritional risk screening; NS: non-statistically significant; RC: radical cystectomy; SMI: skeletal muscle index. 
Supplementary Table 1 (cont'd). Summary of the studies included in this narrative review

\begin{tabular}{|c|c|c|c|c|c|c|}
\hline First author & Year & $\begin{array}{l}\text { Eligible } \\
\text { subjects } \\
\text { (n) }\end{array}$ & $\begin{array}{l}\text { Factor(s) of } \\
\text { nutritional } \\
\text { evaluation }\end{array}$ & Outcome & Results & $\begin{array}{l}\text { Methods of } \\
\text { classification } \\
\text { for } \\
\text { complications }\end{array}$ \\
\hline Wan $^{18}$ & 2014 & 247 & $\begin{array}{l}\text { Albuminemia, } \\
\text { BMI, SMI }\end{array}$ & Complications within 90d & $\begin{array}{l}\text { Logistic regression: Overall complication: } \\
\text { Albuminemia <3.5g/dl (OR 3.63; } \\
95 \% \mathrm{Cl} 1.20-11.00 ; \mathrm{p}=0.0023 \text { ), BMI (NS), } \\
\text { SMI (NS). High-grade complications: } \\
\text { Albuminemia (NS), BMI (NS), SMI } \\
\text { (OR 0.95; } 95 \% \mathrm{Cl} 0.92-0.99 ; \mathrm{p}=0.017 \text { ) }\end{array}$ & Clavien-Dindo \\
\hline Hinata ${ }^{42}$ & 2015 & 730 & $\begin{array}{l}\text { Albuminemia, } \\
\text { BMI }\end{array}$ & Overall survival & $\begin{array}{c}\text { Cox regression: Albuminemia } \\
<3.5 \mathrm{~g} / \mathrm{dl} \text { vs. } 3.5 \mathrm{~g} / \mathrm{dl} \text { (NS). BMl }<22 \mathrm{~kg} / \mathrm{m}^{2} \\
\text { vs. } \geq 22 \mathrm{~kg} / \mathrm{m}^{2} \text { (HR } 1.65 ; \\
95 \% \mathrm{Cl} 1.17-2.33 ; \mathrm{p}=0.004)\end{array}$ & \\
\hline Meyer ${ }^{25}$ & 2015 & 1776 & $\begin{array}{l}\text { Albuminemia, } \\
\text { BMI }\end{array}$ & Wound dehiscence & $\begin{array}{l}\text { Logistic regression: BMI between } 25 \text { and } \\
\left.30 \mathrm{~kg} / \mathrm{m}^{2} \text { (OR 2.1; } 95 \% \mathrm{Cl} 1.1-3.9 ; \mathrm{p}=0.02\right) \\
\text { and } \mathrm{BMI}>30 \mathrm{~kg} / \mathrm{m}^{2} \text { (OR 2.3; } \\
95 \% \mathrm{Cl} 1.3-4.4 ; \mathrm{p}=0.008 \text { ) vs. } \\
\mathrm{BMI}<25 \mathrm{~kg} / \mathrm{m}^{2} \text {. Chi-Square: Albuminemia } \\
\text { (NS), BMI ( } \mathrm{p}=0.015)\end{array}$ & \\
\hline Potretzke $^{43}$ & 2015 & 241 & BMI & $\begin{array}{l}\text { Symptomatic venous } \\
\text { thromboembolic events } \\
\text { within } 90 \mathrm{~d} \text { after } \mathrm{RC}\end{array}$ & $\begin{array}{l}\text { Logistic regression: } \mathrm{BMI} \geq 30 \mathrm{~kg} / \mathrm{m}^{2} \text { vs. } \\
<30 \mathrm{~kg} / \mathrm{m}^{2} \text { (OR } 4.69 ; 95 \% \mathrm{Cl} 1.70-12.92 \text { ) }\end{array}$ & \\
\hline Sun $^{44}$ & 2015 & 2316 & BMI & $\begin{array}{l}\text { Symptomatic venous } \\
\text { thromboembolism within } \\
\text { 90d after RC }\end{array}$ & Logistic regression: BMI $(p=0.0015)$ & \\
\hline Caras $^{27}$ & 2016 & 1,374 & Albuminemia & $\begin{array}{l}\text { 30d complications } \\
\text { (morbidity and mortality) }\end{array}$ & $\begin{array}{l}\text { Logistic regression: Albuminemia } \\
<3.5 \mathrm{~g} / \mathrm{dl} \text { : Morbidity (OR } 1.49 ; \mathrm{p}=0.006 \text { ), } \\
\text { Mortality (NS) }\end{array}$ & \\
\hline $\mathrm{Dabi}^{38}$ & 2016 & 701 & BMI & Cancer-specific mortality & $\begin{array}{c}\text { Cox regression: } \mathrm{BMI}>30 \mathrm{~kg} / \mathrm{m}^{2} \text { vs. } \\
18-25 \mathrm{~kg} / \mathrm{m}^{2} \text { (HR 1.58; } 95 \% \mathrm{Cl} 1.06-2.34 \\
\mathrm{p}=0.02)\end{array}$ & \\
\hline Jensen ${ }^{28}$ & 2016 & 246 & $\begin{array}{l}\text { BMI, nutritional } \\
\text { status }\end{array}$ & Length of hospital stay & $\begin{array}{l}\text { Linear regression: BMI continuously } \\
\text { (NS), Nutritional status (NS) }\end{array}$ & \\
\hline Liu $^{51}$ & 2016 & 296 & $\begin{array}{l}\text { Albuminemia/ } \\
\text { (total } \\
\text { proteinemia/ } \\
\text { albuminemia) } \\
\text { ratio }\end{array}$ & Cancer-specific mortality & $\begin{array}{l}\text { Cox regression: Ratio } \geq 1.6 \text { (HR 0.28; } \\
95 \% \mathrm{Cl} 0.12-0.68 ; p=0.005)\end{array}$ & \\
\hline Movassaghi ${ }^{40}$ & 2016 & 670 & BMI & $\begin{array}{l}\text { Parastomal and incisional } \\
\text { hernia }\end{array}$ & $\begin{array}{c}\text { Cox regression: } \mathrm{BMI} \geq 30 \mathrm{~kg} / \mathrm{m}^{2} \mathrm{vs} \text {. } \\
<30 \mathrm{~kg} / \mathrm{m}^{2} \text { : Parastomal hernia (NS), } \\
\text { Incisional hernia (HR } 2.11 ; 95 \% \mathrm{Cl} 1.26- \\
3.56 ; \mathrm{p}=0.004 \text { ) }\end{array}$ & \\
\hline Osawa ${ }^{19}$ & 2016 & 2,240 & BMI & $\begin{array}{l}\text { 90d complications } \\
\text { (morbidity [Clavien-Dindo } \\
\text { Grade 3-5] and mortality) }\end{array}$ & $\begin{array}{l}\text { Logistic regression: } 90 \mathrm{~d} \text { mortality: } \\
\text { continuously (OR } 1.07 ; 95 \% \mathrm{Cl} 1.02-1.12 \\
\text { p=0.004), } 90 \mathrm{~d} \text { morbidity continuously } \\
\text { (OR } 1.04 ; 95 \% \mathrm{Cl} 1.02-1.07 ; p<0.001)\end{array}$ & Clavien-Dindo \\
\hline
\end{tabular}

AE: adverse event; BMI: body mass indexd: Cl: confidemce interval; d: day(s); HR: hazard ratio; OR: odds ratio; MSKCC: Memorial Sloan Kettering Cancer Classification; Nb: number; NRS: nutritional risk screening; NS: non-statistically significant; RC: radical cystectomy; SMI: skeletal muscle index. 